\title{
Duration of Chemotherapy in Low-versus High-volume Metastatic Cancer: Should There Be a Difference?
}

\author{
Maurie Markman, MD
}

\author{
Address \\ University of Texas M.D. Anderson Cancer Center, Box 121, \\ 1515 Holcombe Boulevard, Houston, TX 77030, USA. \\ E-mail:markman@mdanderson.org \\ Current Oncology Reports 2004, 6:75-76 \\ Current Science Inc. ISSN I523-3790 \\ Copyright (C) 2004 by Current Science Inc.
}

The optimal number of cycles needed for cytotoxic chemotherapy is an important unresolved issue in the management of malignant disease. Randomized trials have shown that a duration of 4 to 6 months of adjuvant chemotherapy for breast cancer brings results that are equivalent with those from regimens of longer duration [1]. Outcomes with 3 cycles of cisplatin, etoposide, and bleomycin are equal to those with 4 cycles in favorable-prognosis disseminated germ cell tumors [2], and 5 years of maintenance therapy for childhood acute lymphoblastic leukemia provides no additional benefit to that achieved with 3 years of such treatment [3]. Shortening the length of therapy has the clear advantage of reducing the time in which patients are required to experience the side effects of antineoplastic drugs, is likely to improve overall quality of life, and has the potential to decrease the cost of a particular treatment regimen.

However, it is also obvious that, for individual tumor types, a minimum number of chemotherapy cycles are required to achieve optimal killing of tumor cells and to attain maximum therapeutic efficacy. For example, a recent report of 10-year survival in a randomized trial examining FEC (fluorouracil, epirubicin, and cyclophosphamide) chemotherapy as an adjuvant treatment for node-positive breast cancer revealed the clear superiority of 6 , compared with only 3 cycles of the antineoplastic program [4].

A particularly troublesome discussion concerns the appropriate number of courses of treatment that should be administered to patients with "larger-volume" versus "smaller-volume" metastatic cancer. Some have suggested that patients with less disease can be given fewer treatment courses. The following factors provide support for this approach:

1. Larger tumor volumes require more treatment cycles to achieve optimal cytotoxicity.
2. With adjuvant treatment programs, a variable proportion $(e g, 30 \%-80 \%)$ of patients will not have any residual cancer. As a result, these individuals cannot benefit from the chemotherapy but will experience the toxicity of the therapeutic regimen.

3. With the superior survival observed in patients having only microscopic disease (eg, adjuvant chemotherapy), the side effects of more prolonged chemotherapy, like that administered to patients with more advanced cancers, cannot be justified.

However, alternative arguments can be advanced to support the conclusion that treatment of micrometastatic, or small-volume macrometastatic, cancer should not differ substantially from treatment of larger-volume metastatic disease.

1. The actual difference between micrometastatic and macrometastatic cancers in the total number of malignant cells present is really quite small. If undetected microscopic disease exists, the cancer is likely to progress and can result in the patient's death.

2. Treating the smallest number of metastatic malignant cells in a reasonably intensive manner may increase the chances for a favorable outcome.

3. If an optimal number of treatment cycles are not administered and the cancer recurs, subsequently administered chemotherapy will almost certainly have less efficacy than that potentially achieved with primary therapy.

4. It is inappropriate to generalize regarding the severity of toxicity experienced by patients receiving additional cycles of chemotherapy (eg, 3 vs 6 or 5 vs 10 courses). Although 3 to 5 cycles of a particular regimen in a specific clinical setting may substantially increase the risk of shortterm (eg, bone marrow suppression) and longterm ( $e g$, neurotoxicity) side effects, this may not be the case with another chemotherapy program in a different malignancy. 
The somewhat controversial results of a trial examining chemotherapy for high-risk, early-stage (stage II, a sub-subset of stage I) ovarian cancer highlights the complexity of these issues. In this study, conducted by the Gynecologic Oncology Group (GOG), patients were randomly assigned to receive either 3 or 6 cycles of a regimen of carboplatin and paclitaxel [5]. (Recently reported data from trials conducted in Europe revealed for the first time the favorable impact on overall survival associated with the administration of adjuvant chemotherapy in this clinical setting [6-8].)

The GOG and other groups consider 6 cycles of carboplatin and a taxane to be the standard of care for chemotherapeutic management of advanced (stage III/IV) ovarian cancer [9]. Thus, it might be asked why one should even consider giving only 3 cycles of chemotherapy for high-risk, early-stage disease. Again, some have theorized that lower metastatic tumor volumes necessitate less chemotherapy. The additional toxicity associated with 3 more cycles of carboplatin plus paclitaxel is a further concern, especially considering that $50 \%$ to $70 \%$ of patients treated for high- risk, early-stage ovarian cancer never experience recurrence of disease. However, this study found a 33\% reduction in the risk of recurrence associated with the administration of 6 cycles of chemotherapy, compared with 3 cycles [5]. (The GOG concluded this was a "negative trial" because the study design required a 50\% reduction in recurrence to consider 6 cycles as superior to 3 cycles.) Although the relatively small sample size $(n=457)$ prevents any definitive statement regarding the impact of the additional 3 chemotherapy cycles on overall survival, a 33\% reduction in the risk of recurrence is notable in a malignancy where the curative potential of treatment at the time of disease progression is recognized to be extremely limited.

Furthermore, one must question the severity of toxicity for the majority of patients with 3 additional cycles of a carboplatin-paclitaxel regimen at doses routinely administered in clinical practice (carboplatin, area under the curve 5-6; paclitaxel, $175 \mathrm{mg} / \mathrm{m}^{2}$ over 3 hours). In fact, patients receiving the initial 3 cycles will have already experienced complete alopecia and the potential for anaphylaxis associated with paclitaxel, and will have revealed their sensitivity for excessive bone marrow suppression and neuropathy. Although individuals showing excessive toxicity after 3 cycles could have their treatment stopped, it must be asked why therapy should be discontinued after 3 cycles in the majority of patients when there is evidence that an additional 3 cycles might reduce the risk of disease recurrence by one third.

It may be reasonable to ask similar questions in other disease settings.

\section{References}

1. Early Breast Cancer Trialists' Collaborative Group: Polychemotherapy for early breast cancer: an overview of the randomised trials. Lancet 1998, 352:930-942.

2. Einhorn LH, Williams SD, Loehrer PJ, et al.: Evaluation of optimal duration of chemotherapy in favorable-prognosis disseminated germ cell tumors: a Southeastern Cancer Study Group protocol. J Clin Oncol 1989, 7:387-391.

3. Miller DR, Leikin SL, Albo VC, et al.: Three versus five years of maintenance therapy are equivalent in childhood acute lymphoblastic leukemia: a report from the Children's Cancer Study Group. J Clin Oncol 1989, 7:316-325.

4. Fumoleau P, Kerbrat P, Romestaing P, et al.: Randomized trial comparing six versus three cycles of epirubicin-based adjuvant chemotherapy in premenopausal, node-positive breast cancer patients: 10-year follow-up results of the French Adjuvant Study Group 01 trial. J Clin Oncol 2003, 21:298-305.

5. Bell J, Brady M, Lage J, et al.: A randomized phase III trial of three versus six cycles of carboplatin and paclitaxel as adjuvant treatment in early stage ovarian epithelial carcinoma: a Gynecologic Oncology Group study [abstract]. Gynecol Oncol 2003, 88:156.

6. Colombo N, Guthrie D, Chiari S, et al.: International Collaborative Ovarian Neoplasm Trial 1: a randomized trial of adjuvant chemotherapy in women with early-stage ovarian cancer. J Natl Cancer Inst 2003, 95:125-132.

7. Trimbos JB, Vergote I, Bolis G, et al.: Impact of adjuvant chemotherapy and surgical staging in early-stage ovarian carcinoma: European Organisation for Research and Treatment of Cancer-Adjuvant ChemoTherapy in Ovarian Neoplasm trial. J Natl Cancer Inst 2003, 95:113-125.

8. Trimbos JB, Parmar M, Vergote I, et al.: International Collaborative Ovarian Neoplasm Trial 1 and Adjuvant ChemoTherapy in Ovarian Neoplasm trial: two parallel randomized phase III trials of adjuvant chemotherapy in patients with early-stage ovarian carcinoma. J Natl Cancer Inst 2003, 95:105-112.

9. Ozols RF, Bundy BN, Greer BE, et al.: Phase III trial of carboplatin and paclitaxel compared with cisplatin and paclitaxel in patients with optimally resected stage III ovarian cancer: a Gynecologic Oncology Group study. J Clin Oncol 2003, 21:3194-3200. 